\title{
ВмJ Global Health Prescribing practices for presumptive TB among private general practitioners in South Africa: a cross-sectional, standardised patient study
}

\author{
Angela Salomon (D) ,,2 Jody Boffa, ${ }^{3,4}$ Sizulu Moyo, ${ }^{5,6}$ Jeremiah Chikovore (D) , ${ }^{5}$ \\ Giorgia Sulis (D) , , 2 Benjamin Daniels (D) , ${ }^{8}$ Ada Kwan, ${ }^{9}$ Tsatsawani Mkhombo, ${ }^{10}$ \\ Sarah Wu, ${ }^{11}$ Madhukar Pai (D) , 2,7 Amrita Daftary (D) ${ }^{12,13}$
}

To cite: Salomon A, Boffa J, Moyo S, et al. Prescribing practices for presumptive TB among private general practitioners in South Africa: a cross-sectional, standardised patient study. BMJ Global Health 2022;7:e007456. doi:10.1136/ bmjgh-2021-007456

Handling editor Seye Abimbola

- Additional supplemental material is published online only. To view, please visit the journal online (http://dx.doi.org/10. 1136/bmjgh-2021-007456)

Received 16 September 2021 Accepted 22 December 2021

Check for updates

(c) Author(s) (or their employer(s)) 2022. Re-use permitted under CC BY. Published by BMJ.

For numbered affiliations see end of article.

Correspondence to Dr Amrita Daftary; adaftary@yorku.ca

\section{ABSTRACT}

Introduction Medicine prescribing practices are integral to quality of care for leading infectious diseases such as tuberculosis (TB). We describe prescribing practices in South Africa's private health sector, where an estimated third of people with TB symptoms first seek care.

Methods Sixteen standardised patients (SPs) presented one of three cases during unannounced visits to private general practitioners (GPs) in Durban and Cape Town: TB symptoms, HIV-positive; TB symptoms, a positive molecular test for TB, HIV-negative; and TB symptoms, history of incomplete TB treatment, HIV-positive. Prescribing practices were recorded in standardised exit interviews and analysed based on their potential to contribute to negative outcomes, including increased healthcare expenditures, antibiotic overuse or misuse, and TB diagnostic delay. Factors associated with antibiotic use were assessed using Poisson regression with a robust variance estimator.

Results Between August 2018 and July 2019, 511 SP visits were completed with $212 \mathrm{GPs}$. In $88.5 \%(95 \% \mathrm{Cl}$ $85.2 \%$ to $91.1 \%$ ) of visits, at least one medicine (median 3) was dispensed or prescribed and most (93\%) were directly dispensed. Antibiotics, which can contribute to TB diagnostic delay, were the most common medicine $(76.5 \%, 95 \% \mathrm{Cl} 71.7 \%$ to $80.7 \%$ of all visits). A majority $(86.1 \%, 95 \% \mathrm{Cl} 82.9 \%$ to $88.5 \%$ ) belonged to the WHO Access group; fluoroquinolones made up $8.8 \%$ (95\% $\mathrm{Cl} 6.3 \%$ to $12.3 \%$ ). Factors associated with antibiotic use included if the SP was asked to follow-up if symptoms persisted ( $\mathrm{RR} 1.14,95 \% \mathrm{Cl} 1.04$ to 1.25 ) and if the SP presented as HIV-positive (RR 1.11, 95\% $\mathrm{Cl} 1.01$ to 1.23 ). An injection was offered in $31.9 \%$ (95\% Cl $27.0 \%$ to $37.2 \%)$ of visits; $92 \%$ were unexplained. Most $(61.8 \%, 95 \% \mathrm{Cl} 60.2 \%$ to $63.3 \%$ ) medicines were not listed on the South African Primary Healthcare Essential Medicines List.

Conclusion Prescribing practices among private GPs for persons presenting with TB-like symptoms in South Africa raise concern about inappropriate antimicrobial use, private healthcare costs and TB diagnostic delay.

\section{BACKGROUND}

Medicines play a crucial role in the delivery of primary healthcare. Their rational use,

\section{Key questions}

What is already known?

- South Africa ranks among the highest tuberculosis (TB) and HIV-associated TB burden countries globally and has a thriving private healthcare sector, where up to a third of people with TB symptoms first seek care.

- Overuse, underuse or misuse of medicines can contribute to adverse drug events, diagnostic delay, antibiotic resistance and growing healthcare expenditures.

- Little is known about prescribing practices in the private sector and how they contribute to quality of TB and HIV care.

What are the new findings?

- When presented with an standardised patient (SP) reporting typical TB symptoms and HIV on probing, private general practitioners (GPs) provided at least one medicine $98.0 \%$ of the time, including an antibiotic $89.6 \%$ of the time.

- Most antibiotics (86.1\%) were from the 'Access' category of the WHO Access, Watch and Reserve framework, indicating lower risk of antibiotic resistance. However, prescription of antibiotics can mask TB symptoms and lead to TB diagnostic delay. The fluoroquinolone class of antibiotics can also contribute to resistance to second-line TB treatment drugs and made up $8.8 \%$ of all antibiotic use in this sector.

- Factors associated with antibiotic use included if there was diagnostic uncertainty, if the SP was asked to return if symptoms persisted, if the SP presented as HIV-positive, and if the GP asked $<3$ TBrelated questions on history.

\section{What do the new findings imply?}

- Prescribing practices among private South African GPs presented with TB-like symptoms raise concerns about inappropriate antimicrobial use, higher healthcare costs and TB diagnostic delay.

- These practices may reflect lack of access to pointof-care testing, diagnostic uncertainty and the need to strengthen private-public referral mechanisms. 
defined by WHO as 'appropriate to (a patient's) clinical needs, in doses that meet their own individual requirements, for an adequate period of time and at the lowest cost to them and their community, ${ }^{1}$ contributes to disease prevention, alleviation and treatment. Their inappropriate use (ie, overuse, underuse or misuse), however, can compromise optimal disease management and trigger avertable complications such as adverse drug events, drug dependence, antimicrobial resistance and patient and health system expenditures. This is especially problematic for severe diseases such as tuberculosis (TB), where inappropriate medicine use can also mask symptoms crucial for diagnosis and lead to delays in the testing, diagnosis and confirmation of TB. ${ }^{2-5}$ Investigation into medicine prescribing practices is thus essential to evaluate overall quality of care in primary care settings.

In South Africa, TB is a leading cause of morbidity and mortality. ${ }^{6}$ Lower respiratory symptoms suggestive of pulmonary TB, among other differential diagnoses, are among the most common clinical presentations in primary care. ${ }^{7}$ Studies in other low-income and middleincome countries demonstrate high rates of inappropriate medicine use, particularly antibiotic use, in primary care settings. ${ }^{89}$ In South Africa's public sector, where most patients with presumptive TB are seen, the rate of inappropriate antibiotic use is estimated to be around $8 \% .{ }^{10}$ However, a third of such patients are estimated to first present to the private sector, ${ }^{11}$ where medicine prescribing practices in response to presentations of TB are understudied. These practices may be further complicated in the South African context by the high underlying prevalence of HIV (19\% in the general adult population and $58 \%$ among people with TB), ${ }^{12}$ which demands patients receive concurrent and integrated attention for not only one but two potentially serious conditions.

Evaluating medicine prescribing practices for TB and HIV-associated TB in the private sector can inform and strengthen national responses to $\mathrm{TB}$, especially given that the private sector has been implicated in TB diagnostic delay. ${ }^{13-15}$ Recently, our team published results of a standardised patient (SP) study that evaluated quality of care for presumptive TB and HIV-associated TB through various indicators including clinical examination, testing and referral practices in the cities of Durban and Cape Town. Over 511 simulated patient interactions with 212 private general practitioners (GPs), we found TB and HIV were ideally managed only $43 \%$ and $41 \%$ of the time, respectively. ${ }^{16}$ In this paper, we describe a subanalysis of medicine prescribing practices among participating GPs to provide further evidence on quality of care gaps and opportunities for enhancing management of TB and HIV-associated TB in South Africa's private sector.

\section{METHODS}

The study used SP methodology, whereby locally recruited and extensively trained staff portray a standardised case presentation during a clinical encounter, which is then documented to measure quality of care. ${ }^{17}$ The SP methodology provides a measure for provider practice that is not subject to typical biases and confounders (eg, patient mix and patient sorting) present in other quality of care methods such as clinical observation, clinician surveys or vignette-based assessments. It has been validated in a number of disease states, including TB. ${ }^{17} 18$

\section{Data collection}

The study was based in South Africa, where the annual incidence rate of TB is 615 per $100000 .{ }^{12}$ Study communities included urban and peri-urban areas of two cities, Durban and Cape Town. Recruitment and data collection methods, as well as the SP training protocol, have been previously published. ${ }^{16}$ Briefly, in each study community, eight SPs were recruited and trained in one of three presentations (table 1). SPs portraying case 1 or 'typical TB' presented with classic TB symptoms and were HIV-positive and antiretroviral therapy (ART)-naïve on probing. SPs

\begin{tabular}{|c|c|c|c|}
\hline $\begin{array}{l}\text { Standardised } \\
\text { patient case }\end{array}$ & Opening statement & Relevant history (if prompted) & $\begin{array}{l}\text { Ideal TB management } \\
\text { strategy* }\end{array}$ \\
\hline $\begin{array}{l}\text { Case 1: 'Basic } \\
\text { TB' }\end{array}$ & $\begin{array}{l}\text { I have a cough and am feeling hot, } \\
\text { and it's not getting better }\end{array}$ & $\begin{array}{l}\text { Cough duration } 2 \text { weeks, experiencing loss of } \\
\text { weight/appetite and night sweats, known HIV+, not } \\
\text { on ART }\end{array}$ & $\begin{array}{l}\text { Offered/sent for any TB } \\
\text { test or referred to public } \\
\text { sector for any reason }\end{array}$ \\
\hline $\begin{array}{l}\text { Case 2: } \\
\text { 'Confirmed TB' }\end{array}$ & $\begin{array}{l}\text { I have a cough that is not getting } \\
\text { better. I have been to a clinic back } \\
\text { home and they gave me some tablets } \\
\text { and took my spit }\end{array}$ & $\begin{array}{l}\text { Carrying GeneXpert pos/Rif inconclusive } \\
\text { laboratory report. Cough duration } 3 \text { weeks, } \\
\text { experiencing loss of weight/appetite and night } \\
\text { sweats, HIV-at last test } 1 \text { year ago }\end{array}$ & \\
\hline $\begin{array}{l}\text { Case 3: } \\
\text { 'Previous TB' }\end{array}$ & $\begin{array}{l}\text { I am suffering from a bad cough. } \\
\text { About a year ago I had got tablets in } \\
\text { the hospital, and it had got better. But } \\
\text { now again I'm having this cough }\end{array}$ & $\begin{array}{l}\text { Cough duration } 2 \text { weeks, experiencing loss of } \\
\text { weight/appetite and night sweats, diagnosed and } \\
\text { treated with TB last year at which time took 3-4 } \\
\text { months TB treatment, known HIV+, not on ART }\end{array}$ & \\
\hline
\end{tabular}

*South African TB guidelines were used as a reference for ideal TB management, which was defined as a verbal or written (1) recommendation for any TB or HIV-related test or (2) referral to the public sector for any reason.

ART, antiretroviral therapy; SPs, standardised patients; TB, tuberculosis. 
portraying case 2 or 'confirmed TB' presented with typical TB symptoms and a positive laboratory report for the detection of Mycobacterium tuberculosis (via Xpert MTB/Rif assay, Cepheid, Sunnyvale, California, USA) and were HIV-negative on probing. SPs portraying case 3 or 'previous TB' presented with typical TB symptoms and a history of incomplete TB treatment, indicating they were HIV-positive and ART-naïve on probing. Training followed an extensive protocol borrowing on previous SP work in TB and tailored to the South African context. ${ }^{17}$ SPs were assessed and confirmed to be in apparent good health to mitigate confounding during clinical encounters.

All GPs registered with the Health Professions Council of South Africa and working within independent private clinics in urban and periurban wards of the two study communities that met the following criteria were included: (1) $\geq 20 \%$ of ward with annual household income $<40000$ South African rand (ZAR; approximately US\$3000), (2) $\geq 1000$ black Africans by subplace (to reflect the local population and minimise SP detection), (3) presence of $>2$ private GPs and (4) within reasonable distance to a public clinic and accessible by public transportation. GPs practising exclusively at private hospitals or providing care to specialised populations (eg, paediatrics, obstetrics) were excluded. Consenting GPs $(n=212)$ received up to three unannounced SPs: the majority (202; 95.3\%) received case 1 and a random sub-sample subsequently received cases 2 and/or 3 in succession to reduce any priming effect. One hundred and one providers $(47.6 \%)$ received all three cases. Immediately following each visit, SPs completed a facilitated interaction survey to record clinical practices, and submitted any artefacts, including medications and prescription notes, given to them by the GP for documentation. To minimise SP detection, each SP portrayed a single case, all SPs presented as walk-in cash-paying patients, no GP working within the same practice received the same case more than once, and a minimum of 2 weeks passed before any GP received a second (or third) case.

\section{Analysis}

This study follows the same analytical approach to interaction surveys as Boffa et al, ${ }^{16}$ where the South African TB guidelines-that do not recommend antibiotic treatment for $\mathrm{TB}$ or HIV-associated TB without a TB test-were used as a reference to evaluate ideal TB and HIV management. ${ }^{19}$ All interaction artefacts (medicines, sputum cups, prescriptions, referral letters, sick notes) were submitted to research staff, anonymized, labelled, photographed and filed according to interaction number. An artefact survey was then completed by trained research staff and double-entered into SurveyCTO (Dobility, Cambridge, Massachusetts, USA). For all prescriptions and medicine products, the following data were documented: active ingredient/s, name (generic or brand), formulation (tablet, syrup, nasal spray, inhaler, cream or other), expiry date, number of pills dispensed and instructions for use, drug classification (drawing on the Anatomical Therapeutic Chemical classification system), ${ }^{8}$ and whether the medicine was on the South Africa Primary Healthcare Essential Medicines List (EML) ${ }^{20}$ Antibiotics were also classified according to the 2019 WHO Access, Watch and Reserve framework based on potential for selecting resistance. $^{21}$ 'Access' antibiotics have activity against a wide range of commonly encountered susceptible pathogens and are thus recommended as the first line of treatment for several infections; 'Watch' antibiotics are broad-spectrum molecules that should be employed with greater caution; 'Reserve' antibiotics are last-resort drugs that should be kept for treatment of confirmed infections due to multidrug-resistant and extremely drug-resistant organisms; and 'Discouraged' antibiotics include fixeddose combinations (FDC) that lack evidence-based indications for use.

We explored prescribing practices using descriptive statistics (proportions, 95\% CIs using bootstrapped estimators of variance, medians and IQR as appropriate) and compared practices between case presentations and study site (Durban vs Cape Town). We examined factors associated with antibiotic use by fitting a series of bivariate Poisson regressions with a robust variance estimator, each adjusted for case presentation and study site because of their potential to contribute to antimicrobial resistance and TB diagnostic delay. ${ }^{2-5}$ However, as TB treatment initiation and management generally occurs in the public sector through the South African national TB programme, we did not analyse TB treatment initiation as an outcome of interest for private GPs. All provider demographics (sex, years in practice, place of training) are included in the bivariate analyses. SPs were considered comparable across SP-provider interactions; hence, we did not adjust for SP-specific demographic variables. All statistical analyses were conducted using Stata V.15.1 (StataCorp).

\section{Patient and public involvement}

This study examines quality of healthcare, in particular quality of medication prescribing practices. The study used SP actors, and participants included physicians. Patients were not recruited or involved in study implementation. Physicians practising in the study communities were invited to attend a free seminar in which study findings were shared and where they could earn continuing medical education credits.

\section{RESULTS}

Between August 2018 and July 2019, 511 interactions (case $1=202$, case $2=157$, case $3=152$ ) were completed with 212 consenting GPs (Durban=96, Cape Town=116). There was a higher proportion of female GPs and those practising in suburbs in Cape Town versus Durban. Other GP characteristics did not significantly differ between SP cases or study sites (table 2). GP participation rate was 
Table 2 Provider characteristics by study site

\begin{tabular}{|c|c|c|c|}
\hline & Overall $(n=511)$ & Durban $(n=220)$ & Cape Town $(n=291)$ \\
\hline \multicolumn{4}{|l|}{ Provider gender, $\mathrm{n}(\%)^{\star}$} \\
\hline Male & $375(73.4)$ & $189(85.9)$ & $186(63.9)$ \\
\hline Female & $136(26.6)$ & $31(14.1)$ & $105(36.1)$ \\
\hline Years in practice, median (IQR) & $25.5(15-35.5)$ & $25(15-33)$ & $26(15-35.5)$ \\
\hline \multicolumn{4}{|l|}{ Location of training, $\mathrm{n}(\%)$} \\
\hline South African Institution & $430(84.3)$ & $178(81.3)$ & $252(86.6)$ \\
\hline International Institution & $80(15.7)$ & $41(18.7)$ & $39(13.4)$ \\
\hline Daily patient load, median (IQR) & $26(15-30)$ & $25(15-33)$ & $20(15-30)$ \\
\hline Consult fee in ZAR†, median (IQR) & $321(280-380)$ & $300(260-350)$ & $350(300-398)$ \\
\hline \multicolumn{4}{|l|}{ Area, $\mathrm{n}(\%)^{*}$} \\
\hline City & $105(20.6)$ & $98(44.6)$ & $7(2.4)$ \\
\hline Township & $119(23.3)$ & $95(43.2)$ & $24(8.3)$ \\
\hline Suburb & 287 (56.2) & $27(12.3)$ & $260(89.4)$ \\
\hline
\end{tabular}

${ }^{*} \mathrm{P}$ value through $\chi^{2}$ test of statistical significance $<0.001$.

†1 ZAR roughly equivalent to US $\$ 0.066$.

$57 \%$. A flow chart depicting consent patterns is included in online supplemental 1.

\section{General patterns of medicines prescribed}

A total of 1576 medicines were entered into the artefact surveys. At least one medicine was provided (prescribed or directly dispensed) in 452 of 511 interactions $(88.5 \%$, $95 \%$ CI $85.2 \%$ to $91.1 \%$ ), with a median of 3 (IQR 2-4) medicines per interaction (table 3$)$. The vast majority $(92.8 \%$,
95\% CI $91.3 \%$ to $94.1 \%$ ) of medicines were dispensed at the point of care (POC) in the sampled clinics; the remainder $(7.2 \%, 95 \%$ CI $5.9 \%$ to $8.7 \%)$ were provided via prescription (table 4). Among medicines directly dispensed, 22 (1.5\%, $95 \%$ CI $0.9 \%$ to $2.4 \%$ ) were unlabelled (across 22 interactions), $3(0.2 \%, 95 \%$ CI $0.0 \%$ to $0.5 \%)$ were expired (across three interactions) and $625(46.5 \%, 95 \%$ CI $40.4 \%$ to $45.1 \%)$ had no visible expiry date (across 145 interactions).

Table 3 Prescribing practices by Interaction and SP case presentation

\begin{tabular}{|c|c|c|c|c|c|c|c|c|}
\hline & \multicolumn{2}{|c|}{ Overall } & \multicolumn{2}{|c|}{ Case 1} & \multicolumn{2}{|c|}{ Case 2} & \multicolumn{2}{|l|}{ Case 3} \\
\hline & $\mathbf{N}$ & $\%(95 \% \mathrm{Cl})$ & $\mathbf{N}$ & $\%(95 \% \mathrm{Cl})$ & $\mathbf{N}$ & $\%(95 \% \mathrm{Cl})$ & $\mathbf{N}$ & $\%(95 \% \mathrm{Cl})$ \\
\hline All interactions & 511 & - & 202 & - & 157 & - & 152 & - \\
\hline No of medicines, median (IQR) & \multicolumn{2}{|c|}{$3(2-4)$} & \multicolumn{2}{|c|}{$4(3-4)$} & \multicolumn{2}{|c|}{$2(0-3)$} & \multicolumn{2}{|l|}{$3(2-4)$} \\
\hline At least one medicine & 452 & 88.5 (85.2 to 91.1$)$ & 198 & 98.0 (95.0 to 99.2) & 113 & $72.0(65.6$ to 77.6$)$ & 141 & 92.8 (88.6 to 95.5$)$ \\
\hline Any antibiotic & 391 & 76.5 (71.7 to 80.7$)$ & 181 & $89.6(84.8$ to 93.0$)$ & 87 & 55.4 (47.7 to 62.9$)$ & 123 & $80.9(72.7$ to 87.1$)$ \\
\hline $\begin{array}{l}\text { Asked about fever or took } \\
\text { temperature }\end{array}$ & 229 & 58.6 (54.0 to 63.0$)$ & 113 & 62.4 (55.8 to 68.6$)$ & 45 & 51.7 (42.8 to 60.6 ) & 71 & 57.7 (47.9 to 70.0$)$ \\
\hline Auscultated lungs & 367 & 93.9 (91.4 to 95.6$)$ & 174 & 96.1 (92.3 to 98.1$)$ & 78 & 89.7 (81.5 to 94.4$)$ & 115 & 93.5 (87.6 to 96.7$)$ \\
\hline $\begin{array}{l}\text { Counselled on finishing } \\
\text { antibiotic course }\end{array}$ & 174 & 44.5 (39.2 to 50.0$)$ & 78 & 43.1 (36.1 to 50.3 ) & 42 & 48.3 (38.7 to 58.0$)$ & 54 & 43.9 (36.9 to 51.1$)$ \\
\hline $\begin{array}{l}\text { Concurrent Ideal TB } \\
\text { Management }^{\star}\end{array}$ & 223 & 57.0 (51.6 to 62.2$)$ & 75 & 41.4 (34.5 to 48.7$)$ & 66 & 75.9 (66.1 to 83.5$)$ & 82 & 66.7 (57.2 to 75.0$)$ \\
\hline Offered injection & 163 & 31.9 (27.0 to 37.2$)$ & 83 & 41.1 (34.6 to 47.9$)$ & 29 & 18.5 (14.0 to 23.9$)$ & 51 & $33.6(26.1$ to 41.9$)$ \\
\hline Unexplained & 150 & 92.0 (89.0 to 94.3$)$ & 77 & 92.8 (86.2 to 96.3$)$ & 24 & $82.8(66.5$ to 92.1$)$ & 49 & 96.1 (88.8 to 98.7 ) \\
\hline 'Influenza/cough' & 8 & 4.9 (2.8 to 8.5$)$ & 5 & $6.0(2.8$ to 12.5$)$ & 3 & 10.3 (3.1 to 2.9$)$ & 0 & - \\
\hline Antibiotic & 4 & 2.5 (1.0 to 6.0$)$ & 1 & $1.2(0.2$ to 8.2$)$ & 2 & 6.9 (1.9 to 2.2$)$ & 1 & $2.0(0.2$ to 15.0$)$ \\
\hline Vitamin BCO & 1 & 0.6 (0.2 to 2.3$)$ & 0 & - & 0 & - & 1 & \\
\hline $\begin{array}{l}\text { Amount paid by SP, median } \\
\text { (range) }\end{array}$ & \multicolumn{2}{|c|}{$330(0-580)$} & \multicolumn{2}{|c|}{$345(100-580)$} & \multicolumn{2}{|c|}{$340(0-500)$} & \multicolumn{2}{|l|}{$320(0-550)$} \\
\hline Medicines dispensed $(n=421)$ & \multicolumn{2}{|c|}{$340(30-580)$} & \multicolumn{2}{|c|}{$340(150-580)$} & \multicolumn{2}{|c|}{$350(30-500)$} & \multicolumn{2}{|l|}{$320(150-550)$} \\
\hline No medicines dispensed $(n=90)$ & \multicolumn{2}{|c|}{$320(0-480)$} & \multicolumn{2}{|c|}{$350(100-450)$} & \multicolumn{2}{|c|}{$310(0-480)$} & \multicolumn{2}{|l|}{$300(0-480)$} \\
\hline
\end{tabular}

*Offered/sent for any TB test or referred to public sector for any reason.

BCO, B Complex; SP, standardised patient; TB, tuberculosis. 
Table 4 Prescribing practices by individual medicine and SP case presentation

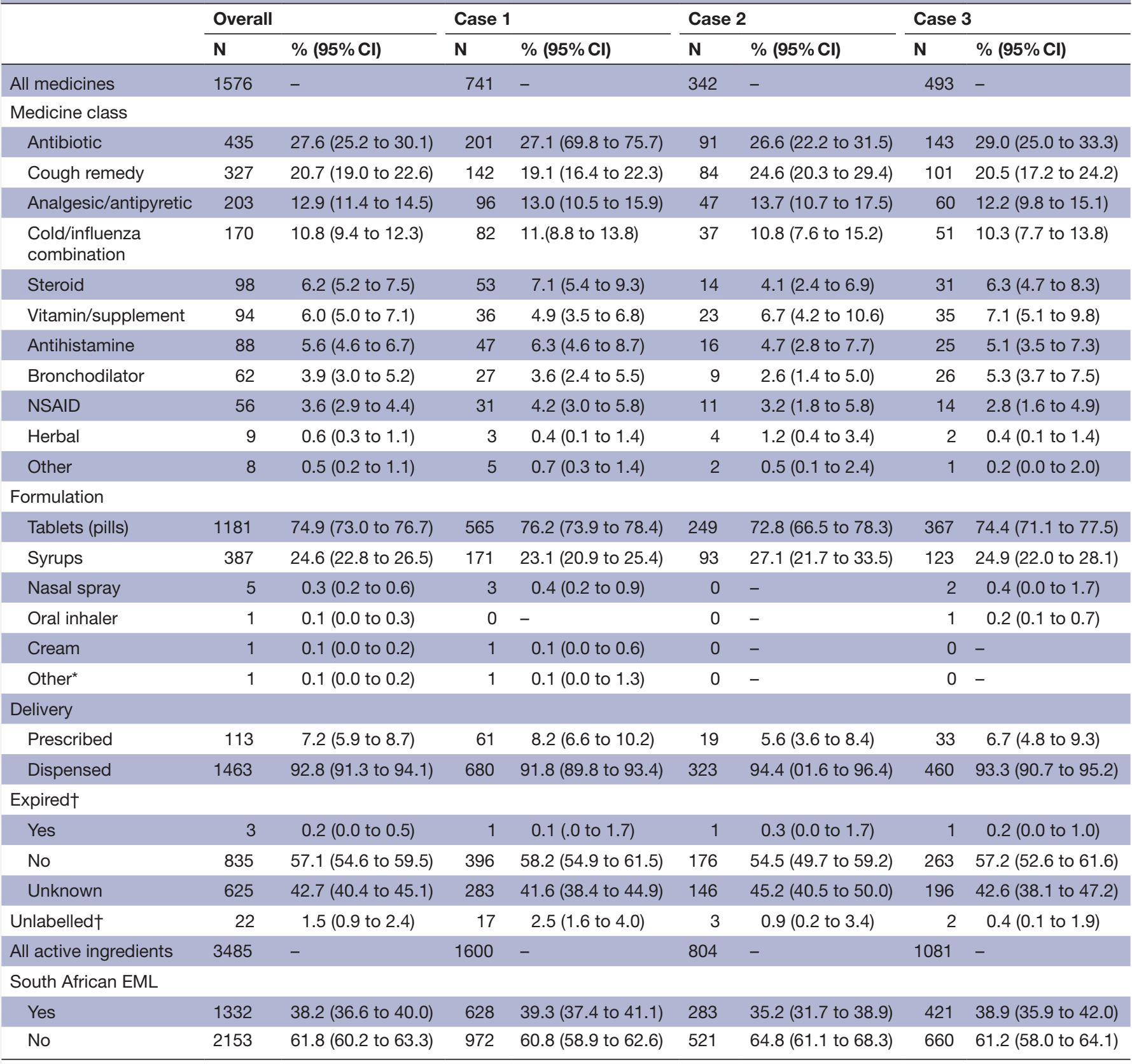

*Ear drops.

†Among medicines directly dispensed $(n=1463)$.

EML, Essential Medicines List; NSAID, Non-Steroidal Anti-Inflammatory Drug; SP, standardised patient.

The most common medicines were antibiotics $(27.6 \%$ of all medicines) provided in 391 of $511(76.5 \%)$ interactions. This was followed by cough remedies $(20.7 \%$ of all medicines), analgesics/antipyretics such as paracetamol and paracetamol/codeine combinations $(12.9 \%$ of all medicines), and cold medicine combinations of paracetamol plus phenylephrine or ephedrine $(10.8 \%$ of all medicines). The most common formulations were tablets $(\mathrm{n}=1181,74.9 \%)$ and syrups $(\mathrm{n}=387,24.6 \%)$, with only one inhaler prescribed (a bronchodilator). The most common active ingredients were paracetamol, cough remedies (ammonium chloride, diphenhydramine and sodium citrate), amoxicillin and theophylline (figure 1); for a list of all active ingredients see online supplemental 2. Although no antiretrovirals were provided, SPs were referred to the public sector or another GP for HIV management and/or ART in $18.4 \%$ of interactions involving an HIV-positive case presentation $(12.4 \%$ of case $1 \%$ and $26.3 \%$ of case 3 ). An injectable medication was offered in 163 interactions $(31.9 \%, 95 \%$ CI $27.0 \%$ to $37.2 \%$ ) (table 3 ). The majority of injections $(92.0 \%$, $95 \%$ CI $89.0 \%$ to $94.3 \%$ ) were not explained to the SP, with the remainder described as 'for influenza/cough' $(4.9 \%, 95 \%$ CI $2.8 \%$ to $8.5 \%)$, antibiotics $(2.5 \%, 95 \%$ CI 


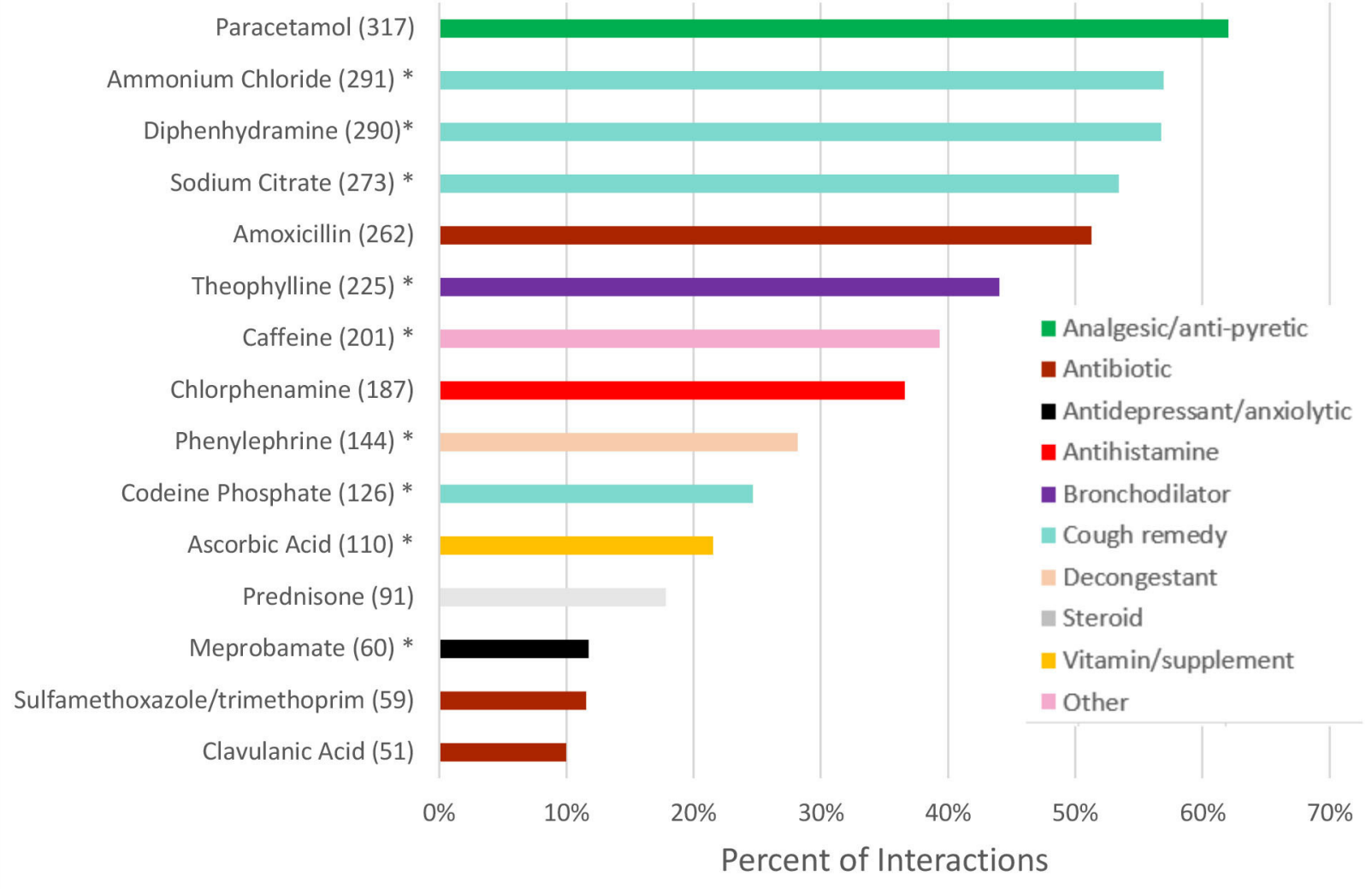

Figure 1 Most commonly prescribed/dispensed active ingredients. The number in brackets is the number of interactions in which that active ingredient was recorded. *Indicates an ingredient not listed on the South African Primary Healthcare Essential Medicines list.

$1.0 \%$ to $6.0 \%)$ or a vitamin B complex $(0.6 \%, 95 \%$ CI $0.2 \%$ to $2.3 \%$ ). All injections were declined by the SP in line with their training.

The majority of medicines $(61.8 \%, 95 \%$ CI $60.2 \%$ to $63.3 \%$ ) were not listed on the South African Primary Healthcare $\mathrm{EML}^{20}$ (table 4). Ingredients not on the EML included those commonly in cough syrups (eg, ammonium chloride, dextromethorphan), bronchodilators (eg, theophylline), non-steroidal anti-inflammatories (eg, diclofenac, indomethacin), analgesics (eg, codeine, oxycodone) and antihistamines (eg, (des)loratadine, levocetirizine). See online supplemental 2 for a full list of concordance of active ingredients with the EML.

Patterns of medicines prescribed varied significantly by case and study site (figure 2). For example, significantly more interactions involving either 'typical TB' (case 1) or 'previous TB' (case 3) resulted in any medicine (Relative risk (RR) $1.3,95 \%$ CI 1.2 to 1.5 ) or offer of an injection (RR 2.1, 95\% CI 1.4 to 2.9) compared with 'confirmed TB' (case 2). Similarly, significantly more interactions in Durban resulted in provision of any medicine ( $R R$ $1.1,95 \%$ CI 1.1 to 1.2 ), an unlabelled medicine (RR 5.2, $95 \%$ CI 1.9 to 13.3), or offer of an injection (RR 10.0, $95 \%$ CI 6.4 to 15.6) compared with those in Cape Town. There were no significant differences between case presentation or study site in the likelihood of prescribing versus directly dispensing a medicine.

\section{Antibiotic prescribing practices}

Of 435 antibiotics provided, penicillins (amoxicillin and amoxicillin/clavulanic acid) were the most common, making up $64.0 \%$ (95\% CI $59.2 \%$ to $68.6 \%$ ), followed by trimethoprim-sulfamethoxazole $(13.6 \%$, 95\% CI $10.7 \%$ to $17.0 \%$ ) and fluoroquinolones (moxifloxacin, levofloxacin, ciprofloxacin) $(8.8 \%, 95 \%$ CI $6.3 \%$ to $12.3 \%)$ (table 5, figure 1 ). The majority of antibiotics $(86.1 \%$, 95\% CI $81.7 \%$ to $89.5 \%$ ) were from the 'Access' group, $56(13.0 \%, 95 \%$ CI $9.6 \%$ to $17.4 \%)$ were from the 'watch' group, and $1(0.2 \%, 95 \%$ CI $0.0 \%$ to $1.9 \%)$ was a 'Discouraged' (FDC, ie, amoxicillin/flucloxacillin) (table 5). No antibiotics were given from the 'Reserve' group. Anti-TB drugs (isoniazid alone, or FDCs of isoniazid, rifampicin, pyrazinamide and ethambutol) were prescribed in three interactions $(0.7 \%$ of all antibiotics, 95\% CI 0.2 to 2.2), all involving the 'confirmed TB' presentation (case 2). Among 391 interactions where an antibiotic was provided, only $57.0 \%$ (95\% CI $52.1 \%$ to $61.9 \%$ ) had concurrent 'Ideal TB Management' (tables 1 and 3 ), and in only $44.5 \%$ (95\% CI $39.2 \%$ to $50.0 \%$ ) the SP was counselled on the importance of completing the 


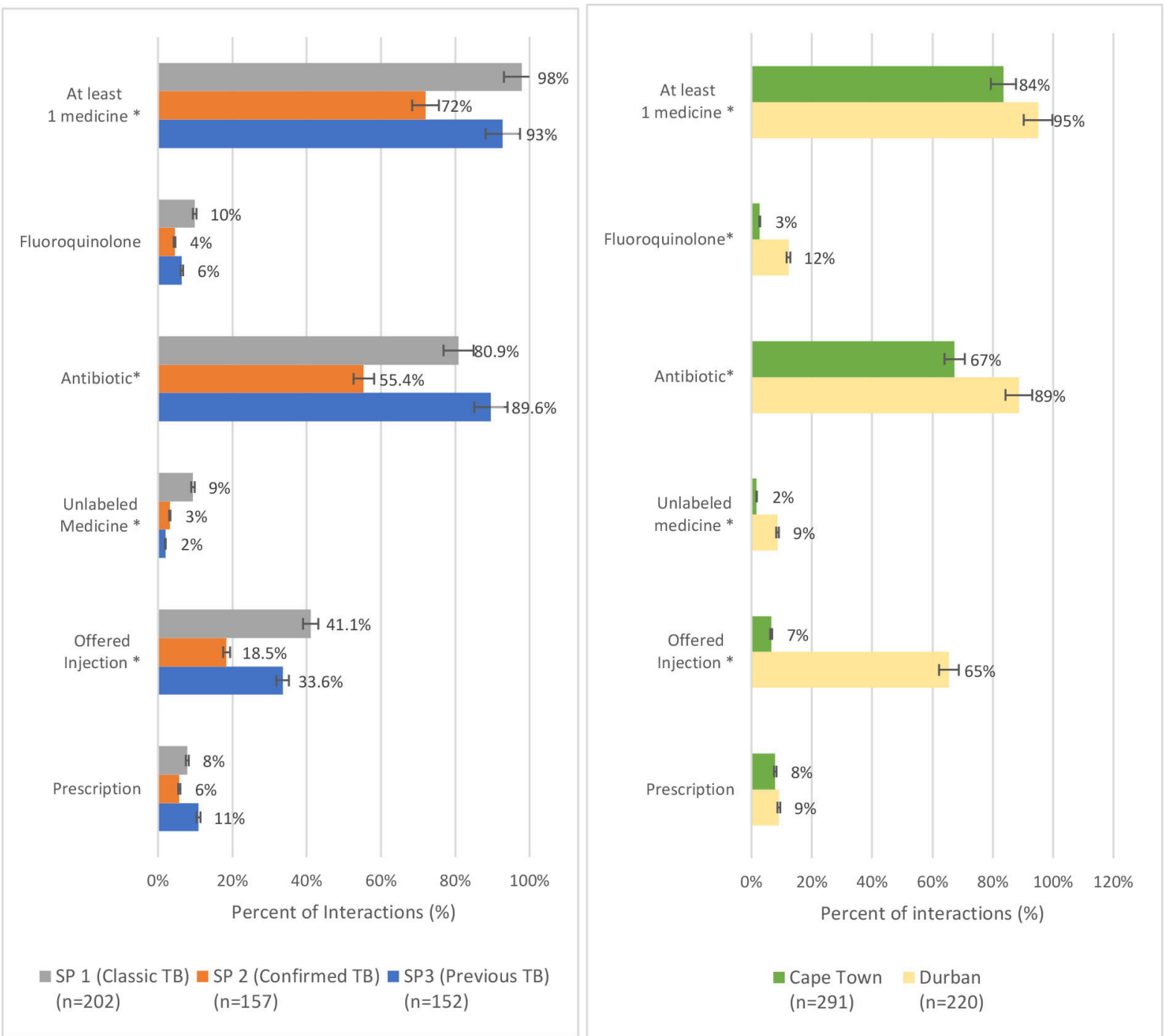

Figure 2 Prescription practices by case and study community. *Significantly different, $p<0.05$. SPs, standardised patients; TB, tuberculosis.

antibiotic course. SPs were determined to be HIV-positive based on history taking in $32.8 \%$ of interactions where sulfamethoxazole/trimethoprim (also known as co-trimoxazole) was provided.

Factors associated with use of any antibiotic included performance of lung auscultation (RR 1.51, 95\% CI 1.15 to 1.98), request for a follow-up visit if symptoms persist (RR 1.14, 95\% CI 1.04 to 1.25 ), or determination of HIVpositivity through history taking (specific to cases 1 and 3, RR 1.11, 95\% CI 1.01 to 1.23) (figure 3). Factors associated with non-use of an antibiotic included presentation of a 'confirmed TB' case (case 2) (RR 0.67, 95\% CI 0.58 to 0.77 ), presentation in Cape Town versus Durban (RR $0.76,95 \%$ CI 0.69 to 0.83 ), concurrent 'ideal TB management' (table 1) (RR 0.82, 95\% CI 0.75 to 0.89 ) and querying $>2$ TB symptoms (from the following four standard screening questions: duration of cough, presence of fever, night sweats, loss of weight or appetite) ${ }^{22}$ (RR 0.90, $95 \%$ CI 0.83 to 0.99$)$. GP-related characteristics such as sex, years in practice $(\leq 25$ vs $>25)$, country of training (South Africa vs international) or minutes spent with SP
( $\leq 10$ vs $>10)$ were not associated with antibiotic use or non-use. In a subanalysis on factors contributing to fluoroquinolone use specifically (among GPs who prescribed any antibiotic), only study site was significantly associated (RR of Cape Town vs Durban $=0.30,95 \%$ CI 0.14 to 0.64 ; online supplemental 3).

\section{Amount paid by SP}

The median fee paid by the SP to the provider was US\$23/ZAR330 (range=US\$0-US\$37/ZAR0-ZAR580). Most $(89 \%)$ of GPs had a single fee regardless of whether medicines were dispensed on site or not, and there was no association between a GP having a two-tiered fee (one price for a consultation; another for consultation + medicines) and the likelihood of a medicine being dispensed $(\mathrm{p}=0.646)$. In 12 interactions involving $11 \mathrm{GPs}$, no fee was charged, and no medicines were directly dispensed. Among all 511 interactions, the average amount paid was US\$4/ZAR67 higher when a medicine was dispensed ( $\mathrm{p}=0.000$ ), and the amount paid was on average US $\$ 0.60$ / ZAR9 (95\% CI US\$0.26 to US\$0.91, ZAE4-ZAR14) higher 
Table 5 Antibiotic-specific practices by individual medicine and SP case presentation

\begin{tabular}{|c|c|c|c|c|c|c|c|c|}
\hline & \multicolumn{2}{|c|}{ Overall } & \multicolumn{2}{|c|}{ Case 1} & \multicolumn{2}{|c|}{ Case 2} & \multicolumn{2}{|c|}{ Case 3} \\
\hline & $\mathbf{N}$ & $\%(95 \% \mathrm{Cl})$ & $\mathbf{N}$ & $\%(95 \% \mathrm{Cl})$ & $\mathbf{N}$ & $\%(95 \% \mathrm{Cl})$ & $\mathbf{N}$ & $\%(95 \% \mathrm{Cl})$ \\
\hline Any antibiotic & 435 & - & 201 & - & 91 & - & 143 & - \\
\hline \multicolumn{9}{|l|}{ AWaRE classification } \\
\hline Access & 374 & 86.1 (82.9 to 88.5 ) & 174 & 87.6 (83.0 to 91.0 ) & 76 & 83.5 (76.4 to 88.8 ) & 121 & 85.3 (77.0 to 91.0$)$ \\
\hline Watch & 56 & 12.8 (10.2 to 16.0$)$ & 24 & 12.1 (8.8 to 16.0$)$ & 11 & 12.1 (7.3 to 19.3$)$ & 21 & 14.7 (9.0 to 23.0)) \\
\hline Reserve & 0 & - & 0 & - & 0 & - & 0 & - \\
\hline Discouraged $^{\star}$ & 1 & 0.2 (0.0 to 2.0$)$ & 1 & 0.5 (0.1 to 1.8$)$ & 0 & - & 0 & - \\
\hline Other† & 4 & 0.9 (0.3 to 2.4$)$ & 0 & - & 4 & $4.4(1.3$ to 14.0$)$ & 0 & - \\
\hline \multicolumn{9}{|l|}{ ATC classification } \\
\hline Penicillin & 275 & 63.2 (58.7 to 67.5 ) & 138 & 68.7 (61.6 to 75.0$)$ & 50 & 54.9 (45.8 to 63.8$)$ & 87 & 60.8 (52.8 to 68.3 ) \\
\hline Sulfonamide $\ddagger$ & 59 & 13.6 (10.7 to 17.0$)$ & 23 & 11.4 (8.6 to 15.0$)$ & 11 & 12.1 (6.9 to 20.2$)$ & 25 & $17.5(12.2$ to 24.4$)$ \\
\hline Fluoroquinolone & 38 & 8.8 (6.3 to 12.3$)$ & 15 & 7.5 (4.2 to 13.1$)$ & 7 & 7.8 (4.1 to 14.2 ) & 16 & 11.3 (7.2 to 17.2$)$ \\
\hline Tetracycline & 26 & 6.0 (4.6 to 7.8 ) & 9 & 4.5 (2.3 to 8.6 ) & 10 & 11.0 (6.5 to 17.9$)$ & 7 & 4.9 (2.3 to 9.9 ) \\
\hline Macrolide & 16 & 3.7 (2.5 to 5.5 ) & 7 & 3.5 (1.5 to 8.0$)$ & 4 & 4.4 (1.6 to 11.9$)$ & 5 & 3.5 (1.5 to 7.8$)$ \\
\hline Cephalosporin & 10 & 2.3 (1.1 to 4.7 ) & 6 & 3.0 (1.5 to 6.1$)$ & 3 & 3.3 (1.5 to 7.2 ) & 1 & 0.7 (0.1 to 4.4$)$ \\
\hline Imidazole§ & 6 & 1.4 (0.6 to 3.3$)$ & 2 & 1.0 (0.3 to 3.3$)$ & 2 & $2.2(0.4$ to 11.0$)$ & 2 & 1.4 (0.4 to 5.5$)$ \\
\hline Antimycobacterial & 3 & 0.7 (0.2 to 2.2 ) & 0 & - & 3 & $3.3(1.7$ to 9.1$)$ & 0 & - \\
\hline Combinations ${ }^{\star} \emptyset$ & 1 & 0.2 (0.0 to 1.5$)$ & 1 & $0.5(0.2$ to 1.6$)$ & 0 & - & 0 & - \\
\hline Unknown & 1 & 0.2 (0.0 to 1.8$)$ & 0 & - & 1 & 1.1 (0.3 to 3.7$)$ & 0 & - \\
\hline
\end{tabular}

*Amoxicillin/flucloxacillin fixed-dose combination.

†Anti-TB treatment $(n=3)$, unknown antibiotic $(n=1)$.

$\ddagger$ Sulfamethoxazole/trimethoprim (co-trimoxazole).

$\S$ Metronidazole.

IDoes not include antimycobacterial combinations.

ATC, Anatomical Therapeutic Chemical; AWaRE, Access, Watch and Reserve; SP, standardised patients; TB, tuberculosis.

for each additional medicine dispensed. The median consultation fee varied by study site (Durban=US $\$ 20$ / ZAR305 vs Cape Town=US\$2/ZAR333, $\mathrm{p}<0.001$ ).

\section{DISCUSSION}

This study adds to a growing body of work on antibiotic use and prescribing practices in response to clinical presentations of presumptive or confirmed TB in South Africa's private sector. ${ }^{23}{ }^{24}$ The study drew on SP methodology, which uniquely assesses real life practices, rather than provider's knowledge or intended practices. The study illuminates how initial management of people presenting to the private sector with TB symptoms could be associated with the inappropriate use of antibiotics and compromise the timely diagnosis of TB. We discuss the implications of the study findings on quality of TB care, antimicrobial stewardship and health expenditures. We also suggest opportunities for engaging private GPs, who remain a crucial entry point into the health system, to bridge the public-private divide and strengthen responses to $\mathrm{TB}$, HIV-associated $\mathrm{TB}$, as well as medicine and antibiotic use more broadly.

GPs offered medicines in nearly $90 \%$ of interactions, and the vast majority were directly dispensed-facilitating potential immediate use-rather than prescribed. Medicine overuse carries the risk of adverse drug events, ${ }^{25}$ and injections, that were offered in nearly a third of interactions, carry additional risks of blood borne infections. ${ }^{26}$ GPs in this study were all registered with HPCSA the regulatory authority that oversees registration and practising of health professionals to ensures patient safety. The common use of medicines may be explained in part by perceived patient expectations and norms about medical service provision, that have been described elsewhere. ${ }^{27-31}$ Injections, for example, are perceived in some settings to have greater efficacy and potency than oral remedies. ${ }^{32-34}$ These perceptions, and GPs' response to them, may also be heightened in the client- and business-centred environment of private practice. Interactions were on average $8.1 \mathrm{~min}$, and interactions that resulted in medicines tended to be shorter than those that did not, although this relationship was not significant ( 6.7 vs $8.2 \mathrm{~min}, \mathrm{p}=0.056$ ). It is possible that GPs who were unable to devote adequate time to counsel on non-pharmaceutical approaches to symptom management were more easily able to resort to pharmaceutical options. ${ }^{29}$ As prescribing practices varied significantly by study site, other sociocultural, geographical and demographic drivers may have also been at play. This may include different systems of referral between public and private sectors, differential access to POC testing, or even different patient expectations based on previous experiences with the healthcare system. 


\section{Factors Associated with Antibiotic Use}

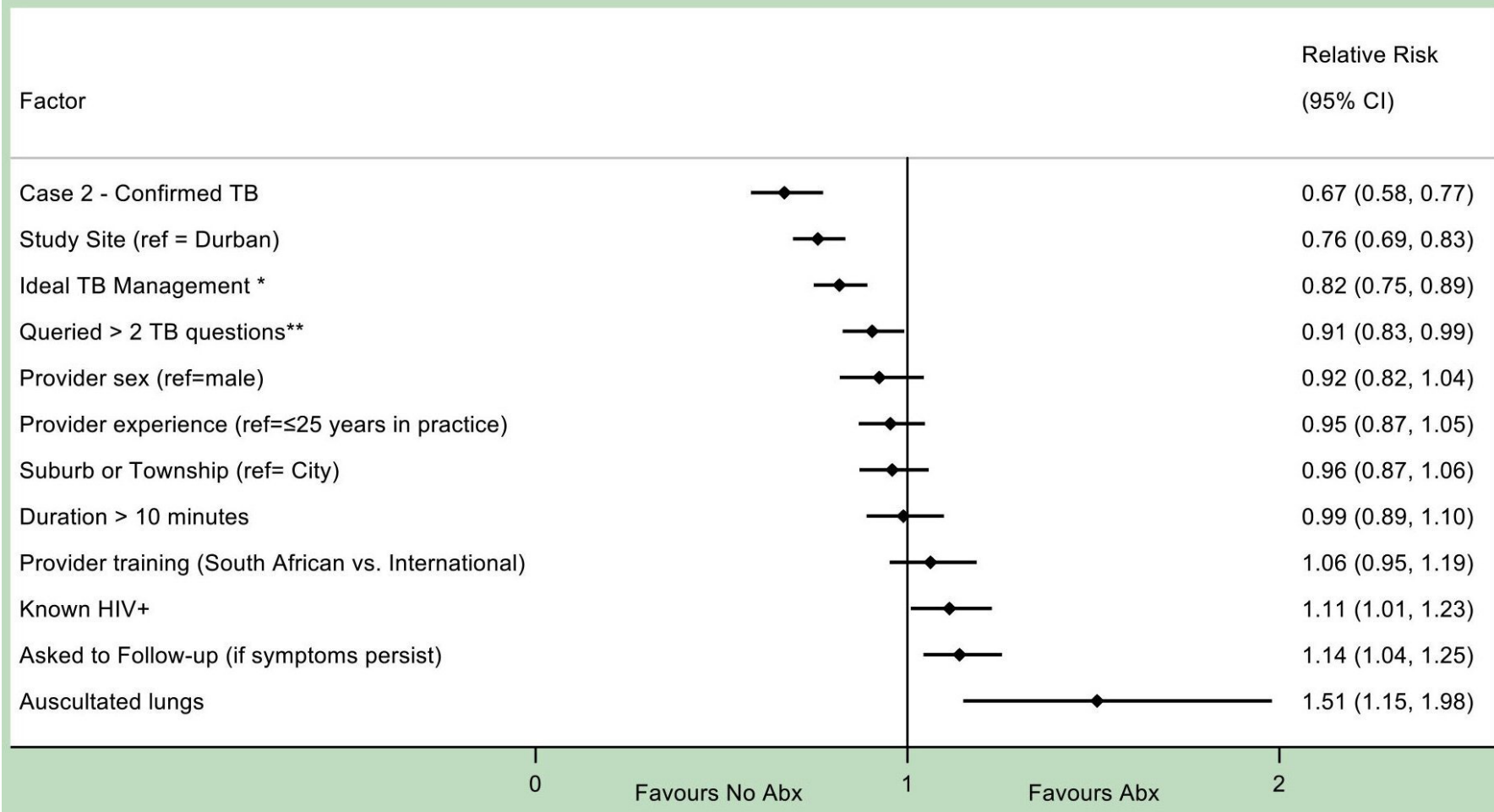

Figure 3 Factors associated with antibiotic dispensing. All analyses adjusted for SP case and study community. *Offered/sent for any TB test or referred to public sector for any reason. ${ }^{*}$ Duration of cough, presence of fever, night sweats, loss of weight or appetite. SPs, standardised patients; TB, tuberculosis.

Among the full range of medicines provided, antibiotics were represented in over $75 \%$ of interactions. This substantiated the results of recent systematic reviews describing high rates of antibiotic prescription in other low-income and middle-income countries including India, China and Kenya. ${ }^{89}$ In this study, most antibiotics $(86 \%)$ were from the 'Access' category, with lower potential of selecting for resistance. Only $12 \%$ of antibiotics were from the 'Watch' category, compared with higher rates observed in similar studies $\left(47.6 \%\right.$ in India, ${ }^{9} 16 \%$ in Ecuador ${ }^{35}$ and Ethiopia, ${ }^{36} 78.4 \%$ in China, ${ }^{37}$ and no antibiotics were dispensed from the 'Reserve' category. The proportion of 'Access'-group antibiotics fell within the range recommended by WHO (ie, at least $60 \%$ of all antibiotics prescribed), thus resulting in a more limited potential for resistance selection. Yet, the overall antibiotic use was still elevated and markedly higher than its use for similar standardised case presentations of TB in the South African public sector $(8 \%) .{ }^{10}$ Most notably, 'Watch' antibiotics were more commonly used than in the public sector (12\% vs $5 \%) .^{38}$ Of these 'Watch' antibiotics, $68 \%$ overall (and $63 \%$ of those dispensed to presentations of confirmed TB, ie, case 2) were fluoroquinolones. Although fluoroquinolone use $(7.7 \%)$ was lower than that observed in other settings such as India $(18 \%),{ }^{39}$ it was higher than in the South African public sector $(0 \%)^{10}$ and, consistent with general medicine prescribing practices, higher in the city of Durban (12\%) than Cape Town (3\%).

These findings have serious potential implications for quality of care in TB and antimicrobial stewardship, that is, optimising antibiotic use to mitigate antimicrobial resistance and infectious disease transmission, and protect patient safety. ${ }^{40}$ Prescription of antibiotics prior to initiating a TB test, and especially prescription of fluoroquinolones, carries the risk of delaying TB diagnoses by masking symptoms and thus delaying test taking and/or diagnostic confirmation. ${ }^{2-5} 4243$ Inappropriate use of fluoroquinolones is further associated with fluoroquinolone-resistance. ${ }^{44} 4546$ There is thus room for building antimicrobial stewardship, now considered integral to health systems strengthening, and to bridging gaps between patient safety and quality of care in primary health settings in the private sector. ${ }^{41}$ However, the use of antibiotics is fraught with complexity. Reports of overuse are balanced by underuse, including in people living with HIV (PLHIV), ${ }^{47}$ for whom antibiotics are a vital line of defence against opportunistic infections. ${ }^{49} 50$ In this study, antibiotics were more commonly used when the standardised case history included a known diagnosis of HIV infection (cases 1 and 3), as was co-trimoxazole more specifically, which is recommended as prophylaxis against opportunistic infections among PLHIV. Postinteraction knowledge surveys conducted alongside our primary research ${ }^{16}$ showed that GPs often considered TB 
in the differential diagnosis (indeed, TB was mentioned in some capacity by the GP in over $80 \%$ of visits), but weighed it against other common diagnoses with similar clinical presentations, including community-acquired pneumonia, acute bronchitis, or acute exacerbations of asthma or chronic obstructive pulmonary disease (see online supplemental 4 for a clinical comparison of differentials of SP case presentations).

In interactions where SPs were asked to return for follow-up, they were also more likely to receive an antibiotic, suggesting empiric antibiotic therapy may have been used as a diagnostic tool. This is common practice in $\mathrm{TB}$ despite the low sensitivity (67\%) and specificity (73\%) for diagnosing pulmonary $\mathrm{TB}$, which is well below international standards. ${ }^{51} 52$ On the other hand, in interactions in which $>2 \mathrm{~TB}$ symptoms were queried, the SP was referred for a TB test and/or to the public sector, or the SP produced a laboratory report indicating GeneXpertconfirmed TB (case 2), antibiotics were significantly less likely to be prescribed. This validates the importance of comprehensive history-taking, and appropriate diagnostic testing in stewarding the appropriate use of therapeutics for respiratory infections. While the South African Standard Treatment Guidelines (2018) recommend empiric antibiotic therapy for other presumed respiratory infections such as pneumonia, they recommend concurrent testing of sputum by GeneXpert to exclude a diagnosis of TB. ${ }^{19} 20$ Enabling use of or access to rapid POC testing such as digital chest X-rays or GeneXpert could mitigate antibiotic misuse and support best practices for TB in initial clinical encounters. However, these tests are also cost prohibitive for small GP practices and cash-paying patients who may not have medical insurance. Evidence from one study suggests the South African public sector initiates TB tests for presentations of basic TB-like symptoms at nearly twice the rate of the private sector $(81 \%$ vs $43 \%) .{ }^{10}$ Hence, there may be value in strengthening partnerships between both sectors as has been recently identified by South Africa's National TB Programme and is envisaged in the National Health Insurance scheme; such programmes are however currently still limited. Other sets of providers can also be engaged in the process of antimicrobial stewardship, including pharmacists and microbiologists. ${ }^{53}$ Innovative investments enabling the utilisation of high-quality POC diagnostics in private health facilities have been successfully implemented in other settings such as India. ${ }^{545}$

Overuse of medicines also has financial implications. In this study, the amount paid by the SP was significantly higher when medicines were dispensed (table 2), and over $60 \%$ of medicines were not part of the South African EML that is designed to support use of the most efficacious and most cost-effective medicine choices. ${ }^{20}$ Although increases in patient costs attributable to medications were marginal (US\$4/ZAR67), and medication dispensation may have been connected to perceptions around quality of care and patient demand, observed prescribing practices may still be contributing to increases in healthcare expenditure. The private sector accounts for $84 \%$ of all pharmaceutical spending (roughly ZAR33.2billion/US\$.9 billion annually), and practices that facilitate use of non-EML medicines could contribute to these growing costs. ${ }^{56}$ Further research is needed to better understand and manage patient expectations, the limitations in which GPs practice, and to strengthen stewardship measures in the private sector to support GP-patient communication, reduce medicine overuse and improve adherence to EML guidance.

This study had several limitations. First, to minimise SP detection, we observed single healthcare encounters and were unable to evaluate potentially complementary (or harmful) practices within follow-up visits. Second, despite rigorous SP training, the novel practices, accents and medical terminology used in real-life interactions may have contributed to recall bias. Thirdly, while SP methods overcome performance and Hawthorne biases common in observational, survey-based or vignette-based quality of care studies, the fact that SPs were actors and did not have overt symptoms of TB on physical examination (eg, fever, wasting, abnormal breath sounds) may have biased GPs away from a diagnosis of TB. However, TB can commonly present without physical symptoms, especially in immunocompromised patients, and thus should remain high on the differential diagnosis (particularly in communities with high rates of TB). Fourthly, although we suggest GPs' practices may have been driven by perceptual and social factors, we did not directly inquire on their rationale or intended duration of recommended prescriptions. This could help to distinguish appropriate medicine use from misuse. For example, it was not possible to distinguish if sulfamethoxazole/trimethoprim (ie, co-trimoxazole preventive therapy) was prescribed to prevent opportunistic infections in SPs who disclosed a positive HIV status (cases 1 and 3) in accordance with national HIV management guidelines, versus used as a broad-spectrum antibiotic to treat an unknown respiratory infection. Finally, our study was conducted prior to the global pandemic of COVID-19 and thus may not represent what would be observed today; in fact, we expect that the challenges we highlighted may be further exacerbated given the similar symptom profile between COVID-19 and TB. ${ }^{57}{ }^{58}$ Similarly, these results and our interpretations may not be generalisable to contexts outside of our high-TB burden, low-middle-income study sites.

\section{CONCLUSION}

This research contributes novel insights into prescribing practices of GPs when presented with symptoms suggestive of TB and alerts us to the potential for these practices to drive health system expenditures, patterns of antibiotic use and TB diagnostic delay. The strategy of 'treatment as diagnosis' may be spurred by diagnostic uncertainty, inaccessibility to POC tests in private facilities, and patient demand for medicines. GPs are, however, key players shaping patients' pathways in the health system. 
Further inquiry into their practices and engaging them in gatekeeping and stewardship measures are likely to have widescale impacts in timely and appropriate management of TB, HIV and related infections.

\section{Author affiliations}

${ }^{1}$ School of Medicine, Queen's University, Kingston, Ontario, Canada

${ }^{2}$ McGill International TB Centre, McGill University, Montréal, Quebec, Canada

${ }^{3}$ Division of Biostatistics and Epidemiology, Stellenbosch University, Stellenbosch, South Africa

${ }^{4}$ Centre for Rural Health, University of KwaZulu-Natal, Durban, South Africa ${ }^{5}$ Human and Social Capabilities Programme, Human Sciences Research Council, Cape Town, South Africa

${ }^{6}$ School of Public Health and Family Medicine, University of Cape Town, Cape Town, South Africa

${ }^{7}$ School of Population and Global Health, McGill University, Montreal, Québec, Canada

${ }^{8} \mathrm{McC}$ Curt School of Public Policy, Georgetown University, Washington, DC, USA

${ }^{9}$ Division of Pulmonary and Critical Care Medicine, University of California School of Medicine, San Francisco, California, USA

${ }^{10}$ Human and Social Capabilities Programme, Human Sciences Research Council, Durban, South Africa

${ }^{11}$ Mailman School of Public Health, Columbia University, New York, New York, USA

${ }^{12}$ School of Global Health \& Dahdaleh Institute of Global Health Research, York University, Toronto, Ontario, Canada

${ }^{13}$ Centre for the AIDS Programme of Research, Durban, KwaZulu-Natal, South Africa

Twitter Angela Salomon @angiesalomon, Jeremiah Chikovore @jerrychikovore, Madhukar Pai @paimadhu and Amrita Daftary @DaftaryAmrita

Acknowledgements The authors are grateful to the participants of this study. We would also like to thank Sean Jooste, Monalisa Jantjies, Linda Thumba and Alicia North at the Human Sciences Research Council, South Africa and Caroline Vadnais at McGill University Health Centre for assistance with research administration.

Contributors AS wrote the first draft. JB, SM, JC, GS, BD, AK, TM, SW, MP and AD reviewed and approved the final version. $A D$ is responsible for the overall content as guarantor.

Funding The study was funded by the Bill \& Melinda Gates Foundation (INV006976)

Disclaimer The funder had no role in the study design, data collection, data analysis, data interpretation or writing of the report.

Competing interests None declared.

Patient consent for publication Not applicable.

Ethics approval Written informed consent was obtained from all study participants. GPs were assured confidentiality, with only aggregate-level outcomes assessed. The study was approved by the ethics committees of the Human Sciences Research Council, South Africa (HSRC Ref\#: 2/18/10/17) and McGill University Health Centre, Canada (MUHC Ref\#: 2018-4390).

Provenance and peer review Not commissioned; externally peer reviewed.

Data availability statement All data relevant to the study are included in the article or uploaded as online supplemental information.

Supplemental material This content has been supplied by the author(s). It has not been vetted by BMJ Publishing Group Limited (BMJ) and may not have been peer-reviewed. Any opinions or recommendations discussed are solely those of the author(s) and are not endorsed by BMJ. BMJ disclaims all liability and responsibility arising from any reliance placed on the content. Where the content includes any translated material, BMJ does not warrant the accuracy and reliability of the translations (including but not limited to local regulations, clinical guidelines, terminology, drug names and drug dosages), and is not responsible for any error and/or omissions arising from translation and adaptation or otherwise.

Open access This is an open access article distributed in accordance with the Creative Commons Attribution 4.0 Unported (CC BY 4.0) license, which permits others to copy, redistribute, remix, transform and build upon this work for any purpose, provided the original work is properly cited, a link to the licence is given, and indication of whether changes were made. See: https://creativecommons.org/ licenses/by/4.0/.
ORCID iDs

Angela Salomon http://orcid.org/0000-0003-1856-953X

Jeremiah Chikovore http://orcid.org/0000-0002-4910-6952

Giorgia Sulis http://orcid.org/0000-0001-6641-0094

Benjamin Daniels http://orcid.org/0000-0001-9652-6653

Madhukar Pai http://orcid.org/0000-0003-3667-4536

Amrita Daftary http://orcid.org/0000-0003-2275-3540

\section{REFERENCES}

1 World Health Organization. The pursuit of responsible use of medicines: sharing and learning from country experiences. Switzerland: World Health Organization, 2012.

2 Craig SE, Bettinson H, Sabin CA, et al. Think TB! Is the diagnosis of pulmonary tuberculosis delayed by the use of antibiotics? Int $J$ Tuberc Lung Dis 2009;13:208-13.

3 Liu Y-C, Huang W-K, Huang T-S, et al. Inappropriate use of antibiotics and the risk for delayed admission and masked diagnosis of infectious diseases. Arch Intern Med 2001:161:2366-70.

4 Golub JE, Bur S, Cronin WA, et al. Impact of empiric antibiotics and chest radiograph on delays in the diagnosis of tuberculosis. Int $J$ Tuberc Lung Dis 2005;9:392-7.

5 Wang M, FitzGerald JM, Richardson K, et al. Is the delay in diagnosis of pulmonary tuberculosis related to exposure to fluoroquinolones or any antibiotic? Int J Tuberc Lung Dis 2011;15:1062-8.

6 Statistics South Africa. Mortality and causes of death in South Africa: findings from death notification: statistics South Africa, 1997.

7 Mash B, Fairall L, Adejayan O, et al. A morbidity survey of South African primary care. PLoS One 2012;7:e32358.

8 Sulis G, Adam P, Nafade V, et al. Antibiotic prescription practices in primary care in low- and middle-income countries: a systematic review and meta-analysis. PLoS Med 2020;17:e1003139.

9 Sulis G, Daniels B, Kwan A, et al. Antibiotic overuse in the primary health care setting: a secondary data analysis of standardised patient studies from India, China and Kenya. BMJ Glob Health 2020;5:e003393.

10 Christian C, Gerdtham U-G, Hompashe D, et al. Measuring quality gaps in TB screening in South Africa using standardised patient analysis. Int J Environ Res Public Health 2018;15:729.

11 Chin DP, Hanson CL. Finding the missing tuberculosis patients. $J$ Infect Dis 2017;216:S675-8.

12 World Health Organization. Global tuberculosis report 2020 Switzerland: World Health Organization, 2020.

13 Van Wyk SS, Enarson DA, Beyers N, et al. Consulting private health care providers aggravates treatment delay in urban South African tuberculosis patients. Int J Tuberc Lung Dis 2011;15:1069-76.

14 Mistry N, Lobo E, Shah S, et al. Pulmonary tuberculosis in Patna, India: durations, delays, and health care seeking behaviour among patients identified through household surveys. J Epidemiol Glob Health 2017;7:241-8.

15 Naidoo P, Theron G, Rangaka MX, et al. The South African tuberculosis care cascade: estimated losses and methodological challenges. J Infect Dis 2017;216:S702-13.

16 Boffa J, Moyo S, Chikovore J, et al. Quality of care for tuberculosis and HIV in the private health sector: a cross-sectional, standardised patient study in South Africa. BMJ Glob Health 2021;6:e005250.

17 Kwan A, Daniels B, Bergkvist S, et al. Use of standardised patients for healthcare quality research in low- and middle-income countries. BMJ Glob Health 2019:4:e001669.

18 Daniels B, Dolinger A, Bedoya G, et al. Use of standardised patients to assess quality of healthcare in Nairobi, Kenya: a pilot, crosssectional study with international comparisons. BMJ Glob Health 2017;2:e000333.

19 South Africa National Department of Health. National tuberculosis management guidelines, 2014.

20 South African National Department of Health. Standard treatment guidelines and essential medicines list for South Africa, primary health care level. National Department of Health Pretoria, 2018.

21 Sharland M, Gandra S, Huttner B, et al. Encouraging AWaReness and Discouraging inappropriate antibiotic use-the new 2019 essential medicines list becomes a global antibiotic stewardship tool. Lancet Infect Dis 2019;19:1278-80.

22 Getahun H, Kittikraisak W, Heilig CM, et al. Development of a standardized screening rule for tuberculosis in people living with HIV in resource-constrained settings: individual participant data metaanalysis of observational studies. PLoS Med 2011;8:e1000391.

23 Ncube NBQ, Solanki GC, Kredo T, et al. Antibiotic prescription patterns of South African general medical practitioners for treatment of acute bronchitis. S Afr Med J 2017;107:119-22. 
24 Lubbe MS, Serfontein JH, Truter I. Antimicrobial prescribing patterns in a group of private primary health care clinics in South Africa. Health SA Gesondheid 2007;12:21-9.

25 Armstrong N, Swinglehurst D. Understanding medical overuse: the case of problematic polypharmacy and the potential of ethnography. Fam Pract 2018;35:526-7.

26 Gore C, Lazarus JV, Peck RJJ, et al. Unnecessary injecting of medicines is still a major public health challenge globally. Trop Med Int Health 2013;18:1157-9.

27 Kohut MR, Keller SC, Linder JA, et al. The inconvincible patient: how clinicians perceive demand for antibiotics in the outpatient setting. Fam Pract 2020;37:276-82.

28 Hoffman D, Botha J, Kleinschmidt I. An assessment of factors influencing the prescribing of antibiotics in acute respiratory illness: a questionnaire study. SA Fam Pract 2003;45:20-4.

29 Manderson L. Prescribing, care and resistance: antibiotic use in urban South Africa. Humanit Soc Sci Commun 2020;7:1-10.

30 Mohamadloo A, Zarein-Dolab S, Ramezankhani A, et al. The main factors of induced demand for medicine prescription: a qualitative study. Iran J Pharm Res 2019;18:479.

31 Lopez C, Sautmann A, Schaner S. Does patient demand contribute to the overuse of prescription drugs? World Bank Group, 2020.

32 Li HK, Agweyu A, English M, et al. An unsupported preference for intravenous antibiotics. PLoS Med 2015;12:e1001825.

33 World Health Organization Safe Injection Global Network. Advocacy booklet. Switzerland: World Health Organization, 2011: 1-25.

34 Chowdhury AKA, Roy T, Faroque ABM, et al. A comprehensive situation assessment of injection practices in primary health care hospitals in Bangladesh. BMC Public Health 2011;11:1-13.

35 Sánchez Choez X, Armijos Acurio ML, Jimbo Sotomayor RE. Appropriateness and adequacy of antibiotic prescription for upper respiratory tract infections in ambulatory health care centers in Ecuador. BMC Pharmacol Toxicol 2018;19:46.

36 Yebyo H, Medhanyie AA, Spigt M, et al. C-Reactive protein pointof-care testing and antibiotic prescribing for acute respiratory tract infections in rural primary health centres of North Ethiopia: a crosssectional study. npj Prim Care Resp Med 2016;26:1-5.

37 Sun Q, Dyar OJ, Zhao L, et al. Overuse of antibiotics for the common cold - attitudes and behaviors among doctors in rural areas of Shandong Province, China. BMC Pharmacol Toxicol 2015;16:6.

38 South Africa National Department of Health. Surveillance for antimicrobial resistance and consumption of antibiotics in South Africa, 2018.

39 Kwan A, Daniels B, Saria V, et al. Variations in the quality of tuberculosis care in urban India: a cross-sectional, standardized patient study in two cities. PLoS Med 2018;15:e1002653.

40 McGregor JC, Fitzpatrick MA, Suda KJ. Expanding antimicrobial stewardship through quality improvement. JAMA Netw Open 2021;4:e211072-e

41 King LM, Fleming-Dutra KE, Hicks LA. Advances in optimizing the prescription of antibiotics in outpatient settings. BMJ 2018;22:k3047.
42 Hogan CA, Puri L, Gore G, et al. Impact of fluoroquinolone treatment on delay of tuberculosis diagnosis: a systematic review and metaanalysis. J Clin Tuberc Other Mycobact Dis 2017;6:1-7.

43 Chen T-C, Lu P-L, Lin C-Y, et al. Fluoroquinolones are associated with delayed treatment and resistance in tuberculosis: a systematic review and meta-analysis. Int $J$ Infect Dis 2011;15:e211-6.

44 Long R, Chong H, Hoeppner V, et al. Empirical treatment of community-acquired pneumonia and the development of fluoroquinolone-resistant tuberculosis. Clin Infect Dis 2009;48:1354-60.

45 Migliori GB, Langendam MW, D'Ambrosio L, et al. Protecting the tuberculosis drug pipeline: stating the case for the rational use of fluoroquinolones. Eur Respir J 2012;40:814-22.

46 Devasia RA, Blackman A, Gebretsadik T, et al. Fluoroquinolone resistance in Mycobacterium tuberculosis: the effect of duration and timing of fluoroquinolone exposure. Am J Respir Crit Care Med 2009;180:365-70.

47 . Leekha S, Terrell Cl, Edson RS, editors. General principles of antimicrobial therapy. Mayo clin proC; 2011: Elsevier.

48 Madeddu G, Laura Fiori M, Stella Mura M. Bacterial communityacquired pneumonia in HIV-infected patients. Curr Opin Pulm Med 2010;16:1-7.

49 Center for Disease Dynamics EP. Lack of access to antibiotics is a major global health challenge. Science Daily 2019.

50 Laxminarayan R, Matsoso P, Pant S, et al. Access to effective antimicrobials: a worldwide challenge. Lancet 2016;387:168-75.

51 McDowell A, Pai M. Treatment as diagnosis and diagnosis as treatment: empirical management of presumptive tuberculosis in India. int $j$ tuberc lung dis 2016;20:536-43.

52 World Health Organization. High priority target product profiles for new tuberculosis diagnostics: report of a consensus meeting, 28-29 April 2014, Geneva, Switzerland. Switzerland: World Health Organization, 2014.

53 Chetty S, Reddy M, Ramsamy Y, et al. Antimicrobial stewardship in South Africa: a scoping review of the published literature. JAC Antimicrob Resist 2019;1:dlz060.

54 Wells WA, Uplekar M, Pai M. Achieving systemic and scalable private sector engagement in tuberculosis care and prevention in Asia. PLoS Med 2015;12:e1001842.

55 Joudyian N, Doshmangir L, Mahdavi M, et al. Public-Private partnerships in primary health care: a scoping review. BMC Health Serv Res 2021;21:1-18.

56 Organisation for Economic Co-operation and Development (OECD). Excessive pricing in pharmaceutical markets - note by South Africa, 2018.

57 Boffa J, Mhlaba T, Sulis G, et al. COVID-19 and tuberculosis in South Africa: a dangerous combination. S Afr Med J 2020;110:341-2.

58 McQuaid CF, McCreesh N, Read JM, et al. The potential impact of COVID-19-related disruption on tuberculosis burden. Eur Respir J 2020;56:2001718. 